\title{
Information Technology for Polypharmacy Appropriateness: What Factors Affect the Use of INTERCheckWEB?
}

\author{
Elisabetta Catrini \\ Universita Carlo Cattaneo \\ Lucrezia Ferrario ( $\sim$ Iferrario@liuc.it) \\ Universita Carlo Cattaneo \\ Antonino Mazzone \\ Aziende Socio Sanitarie Territoriale Ovest Milanese \\ Luca Varalli \\ Aziende Socio Sanitarie Territoriale Ovest Milanese

\section{Federico Gatti} \\ Aziende Socio Sanitarie Territoriale Ovest Milanese

\section{Lorella Cannavacciuolo} \\ Universita degli Studi di Napoli Federico II Dipartimento di Ingegneria Industriale

\section{Gabriella Ferruzzi} \\ Universita degli Studi di Napoli Federico II Dipartimento di Ingegneria Industriale

\section{Emanuela Foglia} \\ Universita Carlo Cattaneo
}

\section{Research article}

Keywords: Technology Acceptance Model, Clinician efficiency, Healthcare technology, Innovation, INTERCheckWEB

Posted Date: September 21st, 2020

DOI: https://doi.org/10.21203/rs.3.rs-51758/v1

License: (c) This work is licensed under a Creative Commons Attribution 4.0 International License. Read Full License 


\section{Abstract}

Background: INTERCheckWEB is one of the most outstanding IT innovative technology that can be implemented at hospital level, supporting the clinicians in the evaluation of the therapy appropriateness for a patient, specially reducing the potentially inappropriate prescriptions for elderly patients in polytherapy. The present paper aims at investigating the relationship between physicians and support decision system in therapy appropriateness for elderly patients in polytherapy, in order to is to assess the factors that can foster or hamper physicians in using INTERCheckWEB for drug prescription.

Methods: A qualitative questionnaire based on a model broadly addressed in literature (Technology Acceptance Model TAM2), was administered to seventy clinicians referring to Internal Medicine wards, of four Italian medium size hospitals. Based on TAM2, authors assess how perceived usefulness, perceived ease of use, image and output quality, affect INTERCheckWeb intention to use. Inferential analyses were conducted to define the main aspects useful to understand the factors impacting on INTERCheckWeb adoption in clinical practice.

Results: The regression analysis reported that image, perceived ease of use and perceived usefulness, as well as the moderator effect of the voluntary use between the perceived usefulness and the intention to use, are the factors that most influenced the use of INTERCheckWEB (Adjusted $R^{2}=0.870$ ).

Conclusions: The analysis has demonstrated that clinicians would effectively use INTERCheckWEB, when available, to identify all the information that could be dangerous for the patient, choosing the proper drug and preventing potential interactions and medical errors.

\section{Background}

Information technologies (ITs) are frequently used to improve the decision-making process in hospitals, especially to gather information in a quick and accurate manner, supporting the clinicians, with a positive impact on the patients [1-4] The use of IT should reduce the complexity of the process management in healthcare and limit clinical errors, thus also improving both the clinical and the safety profiles related to the delivery of care [1-2;5-6]. ITs would offer new opportunities for identifying needs and delivering healthcare services, potentially transforming healthcare organizations in ways that may contribute to achieve healthcare systems goals (high-quality services, efficiency, equity, affordability and accessibility to care) [7].

ITs used in hospital setting, represent a healthcare technology, requiring an in-depth evaluation [8]. Therefore, considering the importance of correctly assessing the impact of innovative healthcare technologies adoption on the existing clinical pathways, it is important to directly involve all the healthcare professionals in the Its' use $[5,9]$. Despite the ITs positive impact on the process efficiency improvement, their adoption is usually related to a professional resilience to change, because of ITs being perceived as disruptive innovations.

Nowadays, INTERCheckWEB is one of the most outstanding IT innovative technology that could be implemented, at hospital level, supporting the clinicians in the evaluation of the therapy appropriateness for a patient, specially reducing the potentially inappropriate prescriptions for elderly patients in polytherapy [10-12], and characterized by a free access solution, without needing an economic investment.

This topic acquires relevance, since literature evidence declared that polypharmacy in the elderly is estimated to be around $40 \%$ of outpatient population, and even higher in selected groups, for example hospitalized patients [13-18]. In order to improve the appropriateness of drugs prescription, the clinicians could benefit from using a Computerized Prescription Support System (CPSS), namely INTERCheckWEB and developed by the Istituto di Ricerche Farmacologiche Mario Negri IRCCS [19]. The INTERCheckWEB aims at storing information about drug-drug interaction (DDI), potentially inappropriate 
medications, anticholinergic burden and dose adjustment in case of renal disease and modality for drug withdrawals. It has a user-friendly interface and can guide clinicians in the evaluation of any possible therapy switches or changes, according to the most recent versions "Beers" and "START and STOPP" criteria [20-21].

Despite the relevance of this tool, no consensus exists, regarding its continuous use, during the phase of drug therapies prescription. Thus, the acceptability of the innovative technology implies a specific validation, concerning the degree of intention to use of this technology, that will be required by the clinicians directly involved in its use [22].

Without the acceptability of the new technology, one cannot expect to achieve any significant result from its development, both in the first phase of its experimental section of the proposal, and in the continuous real practice adoption. Indeed, the resistance to change could lead the clinicians to avoid the adoption of an innovative tool, due to the lack of time concerns in using INTERCheckWEB [23].

\section{Methods}

\section{Development of the theoretical framework}

The study aims at investigating the acceptability of the innovative technology INTERCheckWEB, in case of drugs interaction decisions. This study proposed a theoretical framework that includes the Technology Acceptance Model TAM2 core constructs, useful for predicting the individual's acceptance of ITs, including social and cognitive instrumental factors [24-25].

TAM 2 model was considered because of both its successful use in the healthcare setting, and its parsimony and strong explanatory power [26-28].

Since no evidence on actual use for the innovative technology are registered, in the clinical routine activities, the only intention to use variable was assessed.

Coherently with these premises, the study addressed the following research question: "Which are the key factors determining the intention to use INTERCheckWEB, for clinicians in the clinical practice?"

The deep investigation about the above research question requires the analysis of different independent variables, and their relationship with the acceptability of the innovative technology, measured by the clinicians' intention to use, thus considering the factors suggested by TAM2: perceived usefulness, perceived ease of use, image and output quality.

Based on these arguments, the following hypotheses were, accordingly, developed.

\section{Perceived usefulness}

Perceived usefulness could be defined as "the degree to which a person believes that using a particular system would enhance his or her job performance" [24].

The perceived usefulness of INTERCheckWEB is related to the clinician's effort to improve daily activities, and to the proper use of the tool itself, intended as the simplicity of the operation, aiming at the best quality and safety, for the patient [24, $25,29]$. It emerged a direct and positive relationship between the clinicians' belief of a good use-performance trade-off and a higher perceived usefulness: the greater the instrument perceived usefulness, the greater the clinician's willingness to accept the introduction of INTERCheckWEB in the daily activities.

- HP1: Perceived usefulness has a positive impact on INTERCheckWEB intention to use. 


\section{Perceived ease of use}

Perceived ease of use could be defined as "the level a person believes that using a specific innovative technology would be free of effort" [24]. The perceived ease of use influences the innovation adoption. The ease of use is also influenced by the complexity and compatibility of the innovative system, compared with the current situation $[23,24]$.

In particular, the greater the simplicity of INTERCheckWEB, the greater the perceived ease of use, the greater the willingness of the clinician to accept this instrument.

- HP2: Perceived ease of use has a positive impact on INTERCheckWEB intention to use.

\section{Image}

Image could be defined as "the degree to which the use of an innovation is perceived to enhance a person's status in a social system" [30]. If an innovative technology is strongly recommended by the healthcare organization and the clinician does not embrace this innovation, its image would be negatively compromised. On the other hand, the acceptance of technology and the perceived ease of use of the system would modify the behavior of the clinician, having a positive impact on his image $[25,29]$. In this view, the image of the clinician will be improved as much as the use of INTERCheckWEB is welcome by third parties.

- HP3: Image has a positive impact on INTERCheckWEB intention to use.

\section{Quality Output}

Quality output focuses on the fact that, the information, could be clearer and more detailed, encouraging the clinician to accept its introduction. The strength of the tool is to provide the clinicians with high-quality information, identifying the best way to manage the individuals and organizational aspects of the process. Flexibility is the aspect that best summarizes the tool characteristics, and it is expected to strongly encourage the clinicians to adopt this technology [25]. The greater the flexibility of INTERCheckWEB, meant as the ability of the system to provide information for each investigated element, the greater the quality of the requested output, therefore, the propensity of the clinician to use this instrument.

- HP4: Quality output has a positive impact on INTERCheckWEB intention to use.

Despite the direct relationships between the above-mentioned independent variables and INTERCheckWEB intention to use, the proposed framework also includes two moderator variables (voluntary use and experience).

\section{Voluntary use}

Voluntary use is related to people perceptions. In an environment workplace, the employees' resort to tools because they are influenced by the circumstances [25]. The matter, hereby investigated, is the clinicians' willingness to break down the traditional barriers that may impede the innovation acceptance [31]. Thus, the clinician is more likely to use INTERCheckWEB if the willingness to overcome traditional barriers is higher.

- HP5: Voluntary use positively moderates the relationship between perceived usefulness and INTERCheckWEB intention to use. 


\section{Experience}

Perceived usefulness and intention to use innovative ITs could be higher with increasing experience over time. Furthermore, as individuals gained direct experience with a system, they relied less on social information in defining perceived usefulness. However, they continued to judge a system's usefulness, based on potential status benefits, resulting from its use [25].

Based on the above, the following hypotheses were developed.

- HP6: Experience positively moderates the relationship between perceived usefulness and INTERCheckWEB intention to use.

- HP7: Experience positively moderates the relationship between image and INTERCheckWEB intention to use.

A synthesis of the research framework is proposed in the following Figure 1.

For a more comprehensive framework, a set of control variables was investigated: i) clinician age; ii) clinician working experience; iii) IT attitude (in terms of clinician's attitude to use ITs), and iv) IT skills, in terms of clinicians' capability to use digital instruments.

\section{Study design}

A study design composed of the following three phases was conducted.

\section{Adaptation of the existing scales for a specific healthcare sector questionnaire.}

The questionnaire aimed at gathering clinicians' perceptions with regard to their intention to use the innovative software Intercheckweb, based on specific factors proved to affect this variable. In this view, the questionnaire derived from TAM 2 model, considered all the scales previously published and validated by literature evidence [24-25]. All the scales useful to test the hypotheses concerning the users' acceptability to be supported by personal computers and software, have been selected because of their validation in previous literature, in the healthcare services delivery setting [24-25].

The above questionnaire, based on validated English scales, was translated in the Italian language, in order to avoid comprehension concerns (please see Supplementary File 1).

Before administering the questionnaire, a draft was reviewed by five experts, to verify the coherence and the comprehensibility of the document, especially in the adaptation of some sentences, from other sectors, to the healthcare one.

An Exploratory Factor Analysis (EFA) was carried out, to establish if changes introduced in the adaptation process for healthcare sector had affected the structure of the scales. All the items presenting a factor loading higher than 0.3 were included in the definition variables, because of the maximisation of each construct variance, along one dimension. Furthermore, to assure the reliability of the constructs, the assessment of Cronbach's alpha was implemented. A value greater than 0.7 was assumed, to test items and create the new variables, useful to verify the study hypotheses [32].

\section{Data collection in Italian hospitals wards}

The sample of the study was composed by head physicians and hospital clinical managers of second level $(\mathrm{N}=70)$ referring to the Internal Medicine wards, of four Italian medium size hospitals. 
In June 2019, all the professionals involved in the study completed the questionnaire previously defined, through an interview conducted by a trained interviewer. Besides the personal information related to each respondent (professional role, age, seniority and working experience), the questionnaire was composed by a qualitative section in which a 7-item Likert scale was implemented. In particular, the interview process aimed at getting the clinicians' insight concerning their intention to use INTERCheckWEB and the independent/moderator/control variables previously described that are perceived usefulness, perceived ease of use, image, quality output, voluntary use, as well as IT skills and attitude, as previously described.

\section{Data analysis}

Data derived from the questionnaire were first analysed considering descriptive statistics, frequencies and distributions. Preliminary analyses were performed to ensure no violation of the assumptions of normality, linearity and homoscedasticity.

Furthermore, inferential analyses were conducted.

- Relationships between the variables were investigated, to test the existence of correlations among them. In particular, the "person product-moment" correlation coefficient was assessed, to test the existence of small (from 0.10 to 0.29), medium (from 0.3 to 0.49 ), or large (from 0.5 to 1 ) correlations among variables [33].

- A final investigation of the relationship among the variables, using a hierarchical sequential linear regression model (with enter methodology), was implemented to test the hypotheses, useful in order to establish the impacts of independent variables, and moderators respectively. In particular, the Adjusted $\mathrm{R}^{2}$ was examined, in order to control the explanatory power of each model [34]. This approach allows testing the hypotheses, through incremental models, in order to establish the specific impact of each single input variable, on the dependent variable. The option "exclude case pairwise" was implemented, because was the preferable methodology, for a small sample and avoiding any kind of data exclusion. Three different models were developed, thus defining the influence of the different variables (control, independent and moderator variables), on the dependent variable.

- Model 1: Model composed of only the control variables (clinicians' age, clinicians' working experience, clinicians' IT skills, clinicians' IT attitude).

- Model 2: Model composed of Model 1, with the inclusion of the independent variables (perceived usefulness, perceived ease of use, image and output quality).

- Model 3: Model composed of Model 1 and Model 2, with the inclusion of the moderator variables (moderator effect of voluntary use and experience).

All the statistical analyses were performed using the Statistical Package for Social Science (IBM SPSS Statistics Viewer Version 25).

\section{Results}

\section{The sample under assessment}

The sample was composed of 70 clinicians, most of them being females (63\%). The average age of the clinicians involved was 45.23 years old, with a working experience of 16.14 years. Most part of the sample (74\%) was represented by first level medical managers (versus $26 \%$ composed by second level medical managers).

The sample involved presented a good propensity to use innovative ITs, or PCs. Only 3\% of them declared a lower propensity to use ITs. In general, the clinicians involved could be considered supporter to the innovation, since they declared to be prone to adopt any ITs able to facilitate their daily activities, and, also the therapy prescription. 


\section{The reliability of the scales}

The reliability of the scales, and the related constructs were assessed, proving the freedom of the scale from the random error, and establishing their internal consistency.

Detailed information is shown in Table 1.

Table 1 - Resume of variables

\begin{tabular}{|llllll|}
\hline Construct & $\mathbf{N}$ & $\begin{array}{l}\text { Number of items in the } \\
\text { original scale }\end{array}$ & $\begin{array}{l}\text { Number of validated } \\
\text { items }\end{array}$ & $\begin{array}{l}\text { Explained } \\
\text { variance (\%) }\end{array}$ & $\begin{array}{l}\text { Cronbach's } \\
\text { alpha }\end{array}$ \\
\hline $\begin{array}{l}\text { Perceived } \\
\text { usefulness }\end{array}$ & 70 & 6 & 6 & $98.27 \%$ & 0.982 \\
\hline Easy to use & 70 & 6 & 6 & $86.63 \%$ & 0.969 \\
\hline Voluntary use & 70 & 3 & 2 & $65.85 \%$ & 0.712 \\
\hline Imagine & 70 & 3 & 3 & $76.92 \%$ & 0.850 \\
\hline Output quality & 70 & 2 & 2 & $91.83 \%$ & 0.911 \\
\hline Intention to use & 70 & 2 & 2 & $97.91 \%$ & 0.978 \\
\hline
\end{tabular}

\section{Testing the hypotheses}

Perceived usefulness, perceived ease of use, image and output quality were deeply analysed, in order to define their positive or negative impact on the dependent variable (INTERCheckWEB intention to use). In this view, both the strength and the linear relationship between variables were described.

Table 2 depicts that INTERCheckWEB intention to use was strictly related to a higher ITs attitude $(\beta=0.740, p=0.000)$, higher IT skills $(\beta=0.498, p=0.000)$, a greater perceived usefulness $(\beta=0.887, p=0.000)$ and ease of use $(\beta=0.828, p=0.000)$, and a high-quality output $(\beta=0.757, p=0.000)$. Furthermore, the moderator effect of the voluntary use and experience on perceived usefulness $(\beta=0.441, p=0.000$ and $\beta=0.769, p=0.000$, respectively) presented a positive relationship with the dependent variable.

Table 2 - Correlations among variables 


\begin{tabular}{|c|c|c|c|c|c|c|c|c|c|c|c|c|}
\hline & 1 & 2 & 3 & 4 & 5 & 6 & 7 & 8 & 9 & 10 & 11 & 12 \\
\hline $\begin{array}{l}\text { Intention } \\
\text { to use (1) }\end{array}$ & 1 & & & & & & & & & & & \\
\hline $\begin{array}{l}\text { Clinician } \\
\text { Age (2) }\end{array}$ & -.090 & 1 & & & & & & & & & & \\
\hline $\begin{array}{l}\text { Clinician } \\
\text { Experience } \\
\text { (3) }\end{array}$ & -.104 & $.763^{\star *}$ & 1 & & & & & & & & & \\
\hline $\begin{array}{l}\text { IT attitude } \\
\text { (4) }\end{array}$ & $.740^{\star \star}$ & -.033 & -.110 & 1 & & & & & & & & \\
\hline $\begin{array}{l}\text { IT skills } \\
\text { (5) }\end{array}$ & $.498^{\star *}$ & .066 & -.012 & $.536^{\star *}$ & 1 & & & & & & & \\
\hline $\begin{array}{l}\text { Perceived } \\
\text { usefulness } \\
\text { (6) }\end{array}$ & $.887^{\star \star}$ & -.161 & -.092 & $-.812^{\star \star}$ & $-.506^{\star \star}$ & 1 & & & & & & \\
\hline $\begin{array}{l}\text { Perceived } \\
\text { ease of } \\
\text { use (7) }\end{array}$ & $.828^{\star \star}$ & -.183 & -.137 & $-.777^{\star \star}$ & $-.473^{\star \star}$ & $.827^{\star \star}$ & 1 & & & & & \\
\hline Image (8) & -.087 & -.115 & .101 & -.035 & -.035 & .136 & .121 & 1 & & & & \\
\hline $\begin{array}{l}\text { Output } \\
\text { quality (9) }\end{array}$ & $.757^{\star \star}$ & -.234 & -.202 & $-.608^{\star \star}$ & $-.272^{\star}$ & $.781^{\star \star}$ & $.794^{\star \star}$ & .206 & 1 & & & \\
\hline $\begin{array}{l}\text { Perceived } \\
\text { usefulness } \\
\mathrm{X} \\
\text { voluntary } \\
\text { use (10) }\end{array}$ & $.441^{\star *}$ & -.041 & .057 & -.139 & $-.292^{\star}$ & $.274^{\star}$ & $.299 *$ & -.223 & $.329^{\star \star}$ & 1 & & \\
\hline $\begin{array}{l}\text { Perceived } \\
\text { usefulness } \\
\text { X } \\
\text { experience } \\
\text { (11) }\end{array}$ & $.769^{\star *}$ & -.194 & -.014 & $-.785^{\star *}$ & $-.549^{\star \star}$ & $.888^{\star \star}$ & $.794^{\star \star}$ & .202 & $.704^{\star \star}$ & $.329^{\star *}$ & 1 & \\
\hline $\begin{array}{l}\text { Image X } \\
\text { experience } \\
(12)\end{array}$ & .008 & -.230 & .151 & -.119 & -.197 & .197 & .160 & $.865^{\star \star}$ & .229 & .005 & $.346^{\star \star}$ & 1 \\
\hline
\end{tabular}

Focusing on the relationships between the control and independent variables, the following considerations emerged.

- The higher the ITs skills, the higher the attitude of the clinician to use ITs $(\beta=0.536, p=0.000)$. Both aspects are consequently related to a greater perceived INTERCheckWEB usefulness and ease of use $(p=0.000)$. ITs skills and the attitude of the clinician to use ITs are also related to output quality $(\beta=0.272, p=0.0203$ and $\beta=-0.608, p=0.000$, respectively).

- A strong relationship was reported between the perceived usefulness and the perceived ease of use: the higher the perceived usefulness, the higher the perceived ease of use $(\beta=0.827, p=0.000)$. The perceived usefulness and the perceived ease of use also present a strong relationship with output quality $(p=0.000)$.

A regression analysis was conducted to test the hypotheses. Table 3 reports that no control variables could be considered an antecedent of the intention to use INTERCheckWEB. On the other hand, image, perceived ease of use and perceived 
usefulness, as well as the moderator effect of the voluntary use between the perceived usefulness and the intention to use, are the factors that most influenced the use of INTERCheckWEB (Adjusted R ${ }^{2}=0.870$ ).

Table 3 - Regression model

\begin{tabular}{|c|c|c|c|}
\hline & Model 1 & Model 2 & Model 3 \\
\hline \multicolumn{4}{|l|}{ Control variables } \\
\hline Clinician age & 0.083 & 0.089 & 0.134 \\
\hline Experience & -0.244 & -0.005 & -0.080 \\
\hline IT skills & 0.136 & 0.084 & 0.040 \\
\hline IT attitude & $0.692^{\star}$ & 0.134 & 0.034 \\
\hline \multicolumn{4}{|l|}{ Independent variables } \\
\hline Perceived Usefulness & & $0.647 *$ & $0.735^{\star}$ \\
\hline Perceived Ease of Use & & $0.273^{\star}$ & $0.276^{\star}$ \\
\hline Image & & $-0.229 *$ & $-0.248 *$ \\
\hline Output quality & & 0.161 & 0.091 \\
\hline \multicolumn{4}{|l|}{ Moderators } \\
\hline Perceived Usefulness x Voluntary Use & & & $0.129 *$ \\
\hline Perceived Usefulness x Experience & & & -0.169 \\
\hline Image $x$ Experience & & & 0.110 \\
\hline $\mathrm{R}^{2}$ & 0.598 & 0.876 & 0.891 \\
\hline Adjusted $\mathrm{R}^{2}$ & 0.573 & 0.860 & 0.870 \\
\hline F value & $24.138^{\star}$ & $54.107 *$ & $42.940 *$ \\
\hline$\Delta \mathrm{R}^{2}$ & 0.598 & 0.279 & 0.014 \\
\hline$F\left(\Delta R^{2}\right)$ & $24.138^{\star}$ & $34.426 *$ & 2.502 \\
\hline
\end{tabular}

\section{Discussion}

The presence of comorbidities and multiple chronic diseases, and the related prescription of complex medications, are becoming typical problems, in particular in medical wards, and present many professional challenges [35-36]. The concept of "polypharmacy" and high dosage frequency are both associated with high rates of adverse drug reactions, poor adherence, and frequent hospitalization [37-40]. Thus, any strategy able to prevent potentially severe drug-drug 
interactions, and capable to support the clinicians in prescribing the right therapy are currently playing a crucial role in advancing management of chronic illnesses and polypharmacy [41-44]. In this view, the innovative instrument INTERCheckWEB could help the clinicians in choosing the right drug and the right dosage, when managing complex patients affected by several concomitant diseases, thus preventing the development of drug-drug interactions.

The literature evidence available on the topic has demonstrated the efficacy profiles of INTERCheckWEB, in terms of a significant reduction in potentially inappropriate medications and new-onset potentially severe drug-drug interactions, thus being an important strategy for optimizing medication prescription for elderly patients [16]. Given the proven efficacy, since INTERCheckWEB is a free and open access tool and grounds its use only on the Internet connection presence, it does not report any economic or organisational concerns. In this view, no inter-operability problems emerged in the routinely adoption of INTERCheckWEB. The only issue that required an in-depth evaluation from an organisational point of view could be the resilience to effectively adopt the innovative IT, thus being the only potential barrier in limiting the diffusion and consequent implementation of INTERCheckWEB.

The study aimed at showing the existence of possible determinants and predictors of the innovative instrument' intention to use, useful to prevent potential errors in prescribing drugs. The analyses demonstrated that, at least in the investigated setting, INTERCheckWEB intention to use is strictly dependent from the usefulness and the ease of use perceived by the clinician.

Figure 2 shows the main findings after testing the hypotheses.

In particular, the adoption of INTERCheckWEB could also present a relevant medical-legal impact: since it is an updated instrument, grounded on the most recently published clinical evidence, and being capable to reduce potential prescribing errors, its adoption could help clinicians in making evidence-based choices.

It should be noted that a negative relationship emerged between image and INTERCheckWEB intention to use. Clinicians would not utilize INTERCheckWEB for enhancing their "status symbol", but they voluntarily would use the innovative technology only if they are able to find a real usefulness and advantage for improving patients' health results.

The lack of statistical significance with regard to the output quality, could be related to the fact that not all the clinicians were aware of the existence of INTERCheckWEB.

From an organizational point of view, based on the above considerations, the institution of communication campaigns to reveal the existence of INTERCheckWEB could represent a useful strategy to maximize its implementation in the clinical practice.

Despite TAM2 is usually tested using Structure Equation Modelling (SEM), the above-mentioned results relied on the implementation of traditionally regression analysis, in order to verify the robustness of the selected variables using a different quantitative model approach. It could be a topic of further researches, to study the update of the results, through the implementation of SEM technique, in order to define if any important changes occur in the definition of the predictors of INTERCheckWEB intention to use.

Another topic for further researches could be the economic evaluation of the ceasing costs related to a reduction in drugs' prescription, that could consequently reduce the economic burden related to the management of a chronic and elder patient [45].

\section{Conclusions}

The analysis has demonstrated that clinicians would effectively use INTERCheckWEB, when available, to identify, timely, and in an aggregate manner, all the information that could be dangerous for the patient, thus choosing the proper drug and 
preventing potential interactions and medical errors, also personalizing treatment for elderly patients [46].

\section{Abbreviation}

DDI Drug-drug interaction

EFA Explanatory Factor Analysis

IT(s) Information Technology (ies)

TAM Technology Acceptance Model

\section{Declarations}

Ethics approval and consent to participate: not applicable, since the research investigated only the intention to use INTERCheckWEB, without collecting human and sensible data. In particular, all the clinicians involved voluntary completed the questionnaire. According to the above, and after having informed the Healthcare Directorates of the hospitals involved (ASST Ovest Milanese, composed of four different hospitals - Legnano Hospital, Magenta Hospital, Abbiategrasso Hospital and Cuggiono Hospital), the Healthcare Directorates approved the clinicians' perceptions data collection, according to the study protocol number 5135 (Class 03.08.01) dated $8^{\text {th }}$ February 2019.

Consent for publication: All the clinicians involved were aware that the present research activity had the main aim the publication of the results, and they gave their consents in disseminating their perceptions, in an aggregated and anonymous manner. The approval and the consent for publication was also given by the Healthcare Directorates(ASST Ovest Milanese, composed of four different hospitals - Legnano Hospital, Magenta Hospital, Abbiategrasso Hospital and Cuggiono Hospital), , according to the study protocol number 5135 (Class 03.08.01) dated $8^{\text {th }}$ February 2019.

Availability of data and materials: The datasets used and/or analysed during the current study are available from the corresponding author on reasonable request.

Competing Interest: The authors declare that they have no competing interests.

Funding: none

Authors' contributions: EF, AM and EV conceived the study. EC, LF and FG collected and analyzed data. EF, EC and LF wrote the paper. LC and GF critically reviewed the paper. All authors read and approved the final manuscript.

Acknowledgement: The Authors of the present paper would like to sincerely thank all the healthcare professionals involved in the administration of the questionnaire, useful to retrieve important data for the achievement of the objectives.

Furthermore, the Authors would like to also thank Prof. Luca Pasina (Istituto di Ricerche Farmacologiche Mario Negri), who critically revised the contents.

\section{References}

1. Lenz R, Kuhn K.A. Towards a continuous evolution and adaptation of information systems in healthcare. Int J Med Inform. 2004; 73:(1): 75-89.

2. Berg M. Implementing information systems in health care organizations: myths and challenges. Int J Med Inform. 2001; 64(2-3): 143-156.

3. Øvretveit J., Scott T., Rundall T. G., et al. Improving quality through effective implementation of information technology in healthcare. Int J Qual Health Care. 2007; 19(5): 259-266.

Page $11 / 15$ 
4. Ossebaard, H. C., \& Van Gemert-Pijnen, L. eHealth and quality in health care: implementation time. Int J Qual Health Care. 2016: 28(3): 415-419.

5. LeRouge C, Mantzana V and Wilson EV. Healthcare information systems research, revelations and visions. European Journal of Information Systems. 2007; 16: 669-671.

6. Risør, B. W., Lisby, M., \& Sørensen, J..Complex automated medication systems reduce medication administration errors in a Danish acute medical unit. Int J Qual Health Care. 2018; 30(6): 457-465.

7. Braithwaite, J., Mannion, R., Matsuyama, Y., et al. The future of health systems to 2030: a roadmap for global progress and sustainability. Int J Qual Health Care. 2018; 30(10): 823-831.

8. World Health Organisation. Resolution on health technologies - WHA60.29. 2007. Available at: https://www.who.int/healthsystems/WHA60_29.pdf?ua=1

9. Mantzana V, Themistocleous M, Irani Z and Morabito V. Identifying healthcare actors involved in the adoption of information systems. European Journal of Information Systems, 2007; 16(1): 91-102.

10. Gujjarlamudi HB. Polytherapy and drug interactions in elderly. J Midlife Health. 2016; 7(3): 105-107.

11. Magnani L. Potentially Prescriptions Inappropriate (PPIs) in elderly patients in polytherapy: structured discussion on the effect of medication therapy review using evaluation criteria listed in literature on the administrative health care database. Clin Trials Degener Dis 2018; 3: 22-6.

12. Vasilevskis, E.E., Shah, A.S., Hollingsworth, E.K. et al. A patient-centered deprescribing intervention for hospitalized older patients with polypharmacy: rationale and design of the Shed-MEDS randomized controlled trial. BMC Health Serv Res 2019: 19, 165

13. Banerjee A, Mbamalu D, Ebrahimi S, et al. The prevalence of polypharmacy in elderly attenders to an emergency department - a problem with a need for an effective solution. Int J Emerg Med. 2011; 4: 22.

14. Maher RL, Hanlon JT, Hajjar ER. Clinical Consequences of Polypharmacy in Elderly. Expert Opin Drug Saf. 2014; 13(1): 57-65.

15. Olmos R, Garcia O, Velasco J, de la Rubia A. Prevalence of polypharmacy in older hospitalised patients. Eur J Hosp Pharm 2012; 19: 242-243.

16. Slabaugh SL, Maio V, Templin M, Abouzaid S. Prevalence and risk of polypharmacy among the elderly in an outpatient setting: a retrospective cohort study in the Emilia-Romagna region, Italy. Drugs Aging. 2010;27(12):1019-28

17. Kim, J., \& Parish, A. L. Polypharmacy and medication management in older adults. Nurs Clin North Am. 2017; 52(3):457-46

18. Wastesson, J. W., Morin, L., Laroche, M. L., \& Johnell, K. How chronic is polypharmacy in old age? A longitudinal nationwide cohort study. J Am Geriatr Soc. 2019; 67(3): 455-462.

19. Ghibelli S, Marengoni A, Djade CD, et al. Prevention of inappropriate prescribing in hospitalized older patients using a computerized prescription support system (INTERcheck(®)). Drugs Aging. 2013;30(10):821-8.

20. O'Mahony D, O'Sullivan D, Byrne S, et al. STOPP/START criteria for potentially inappropriate prescribing in older people: version 2. Age Ageing. 2015;44(2):213-8.

21. By the 2019 American Geriatrics Society Beers Criteria ${ }^{\circledR}$ Update Expert Panel. American Geriatrics Society 2019 Updated AGS Beers Criteria ${ }^{\circledR}$ for Potentially Inappropriate Medication Use in Older Adults. J Am Geriatr Soc. 2019;67(4):674-694

22. de Veer, A.J.E., Peeters, J.M., Brabers, A.E. et al. Determinants of the intention to use e-Health by community dwelling older people. BMC Health Serv Res 2015: 15, 103.

23. Bhattacherjee A. and Hikmet N. Physicians' resistance toward healthcare information technology: a theoretical model and empirical test. European Journal of Information Systems, 2007; 16(6): 725-737. 
24. Davis FD. Perceived Usefulness, Perceived Ease of Use, and User Acceptance of Information Technology. Management Information Systems, 1989; 13(3): 319-340.

25. Venkatesh V, Davis FD. A Theoretical Extension of the Technology Acceptance Model: Four Longitudinal Field Studies. Management Science, 2000; 46(2): 186-204.

26. Wu, I.L., J.Y. Li and C.Y. Fu. The adoption of mobile healthcare by hospital's professionals: An integrative perspective. Decis. Support Syst. 2011; 51: 587-596.

27. Dunnebeil, S., A. Sunyaev, I. Blohm, J.M. et al. Determinants of physicians' technology acceptance for e-health in ambulatory care. Int. J. Med. Inform. 2012; 81: 746-760.

28. Xue, L., C.C. Yen, L. Chang, H.C. et al. An exploratory study of ageing women's perception on access to health informatics via a mobile phone-based intervention. Int. J. Med. Inform. 2012; 81: 637-648.

29. Holden JR, Karsh BT. The Technology Acceptance Model: Its past and its future in health care. Journal of Biomedical Informatics, 2010; 43(1): 159-172.

30. Moore, G. C., I. Benbasat. Development of an instrument to measure the perceptions of adopting an information technology innovation. Information Systems Research, 1991; 2: 192-222.

31. Yarbrough AK, Smith TB. Technology Acceptance among Physicians. Med Care Res Rev. 2007;64(6):650-72

32. Nunnally J.C., Bernstein I.H., Berge J.M.T. Psychometric theory (Vol. 226), New York (NY): McGraw-Hill, 1967.

33. Cohen, J. Statistical power analysis for the behavioral sciences. 1988.

34. Tabachnick, BG, Fidell, LS. Using multivariate statistics,2007: 5th edn.

35. Ritchie C. Health Care quality and multimorbidity: the jury is still out. Med Care. 2007; 45(6): 477-479

36. Crowley, E.K., Sallevelt, B.T.G.M., Huibers, C.J. et al. Intervention protocol: OPtimising thERapy to prevent avoidable hospital Admission in the Multi-morbid elderly (OPERAM): a structured medication review with support of a computerised decision support system. BMC Health Serv Res 2020; 20, 220

37. Vik, S. A., Maxwell, C. J. \& Hogan, D. B. Measurement, correlates, and health outcomes of medication adherence among seniors. Ann Pharmacother. 2004; 38: 303-312

38. Calderón-Larrañaga A., Poblador-Plou B, González-Rubio F, et al. Multimorbidity, polypharmacy, referrals, and adverse drug events: are we doing things well? Br J Gen Pract. 2012; 62: e821-826.

39. Toh MR, Teo V, Kwan WH, et a. Association between number of doses per day, number of medications and patient's non-compliance, and frequency of readmissions in a multi-ethnic Asian population. Prev Med Reports. 2014; 1: 43-47

40. Rodríguez Pérez A., Rocío Alfaro Lara E., Nieto Martín MD, et al. Deprescribing in Patients With Multimorbidity: A Necessary Process. Eur J Intern Med. 2015;26(7):e18-9.

41. Johansson, T., Abuzahra, M. E., Keller, S et al. Impact of strategies to reduce polypharmacy on clinically relevant endpoints: a systematic review and meta-analysis. Br J Clin Pharmacol. 2016;82(2):532-48.

42. Guo JY, Chou Y, Pu C. Effect of continuity of care on drug-drug interactions. Med Care. 2017; 55(8): 744-751

43. Reeve E., Thompson W., Farrel B., Deprescribing: A Narrative Review of the Evidence and Practical Recommendations for Recognizing Opportunities and Taking Action. Eur J Intern Med 2017;38:3-11

44. Linsky, A., Meterko, M., Stolzmann, K. et al. Supporting medication discontinuation: provider preferences for interventions to facilitate deprescribing. BMC Health Serv Res 2017; 17, 447

45. Hyttinen V, Jyrkkä J, Valtonen H. A Systematic Review of the Impact of Potentially Inappropriate Medication on Health Care Utilization and Costs Among Older Adults. Med Care. 2016;54(10):950-64

46. Cenci C. Narrative Medicine and the Personalisation of Treatment for Elderly Patients. Eur J Intern Med. 2016;32:22-5.

\section{Figures}




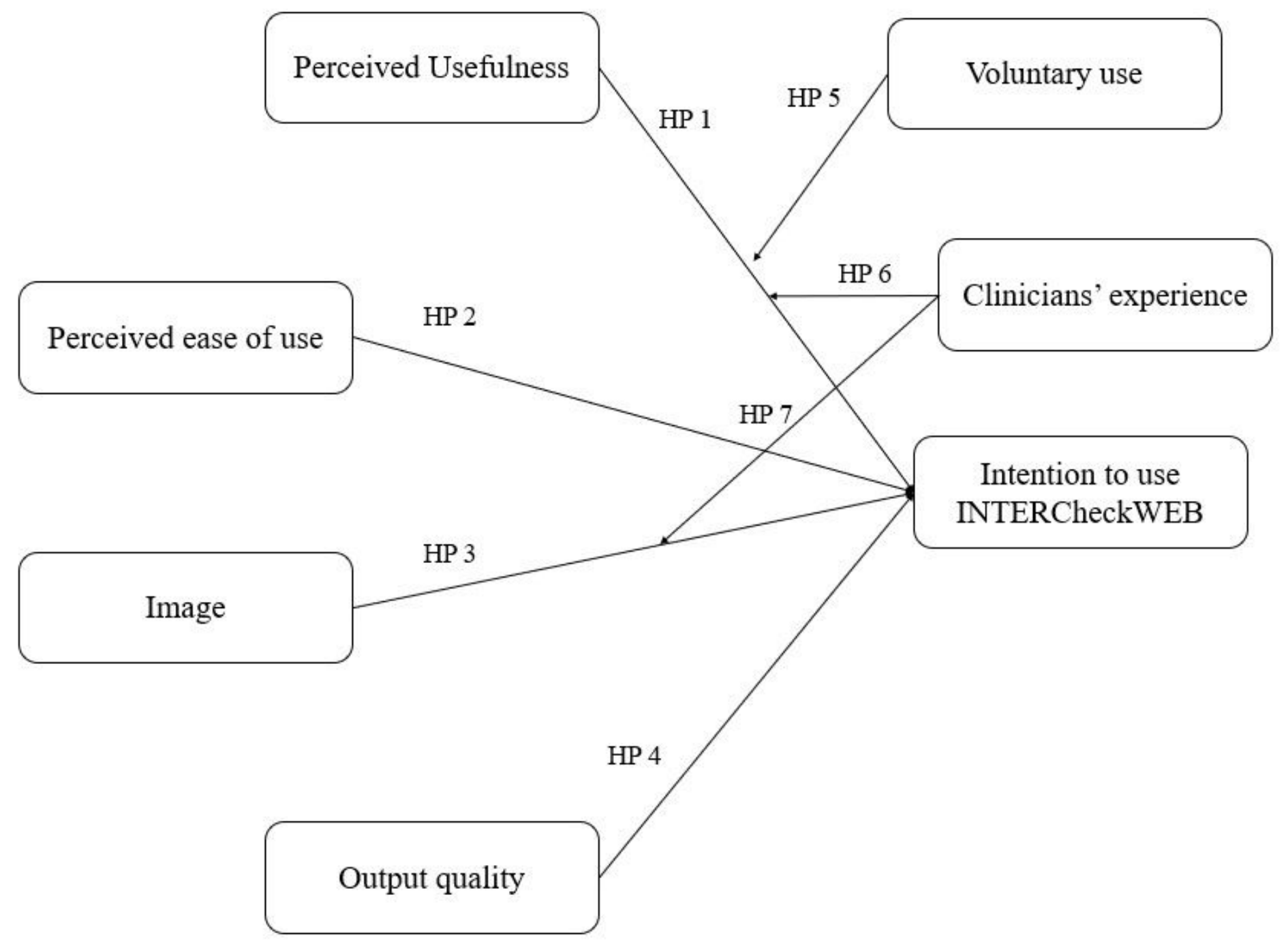

Figure 1

Framework 


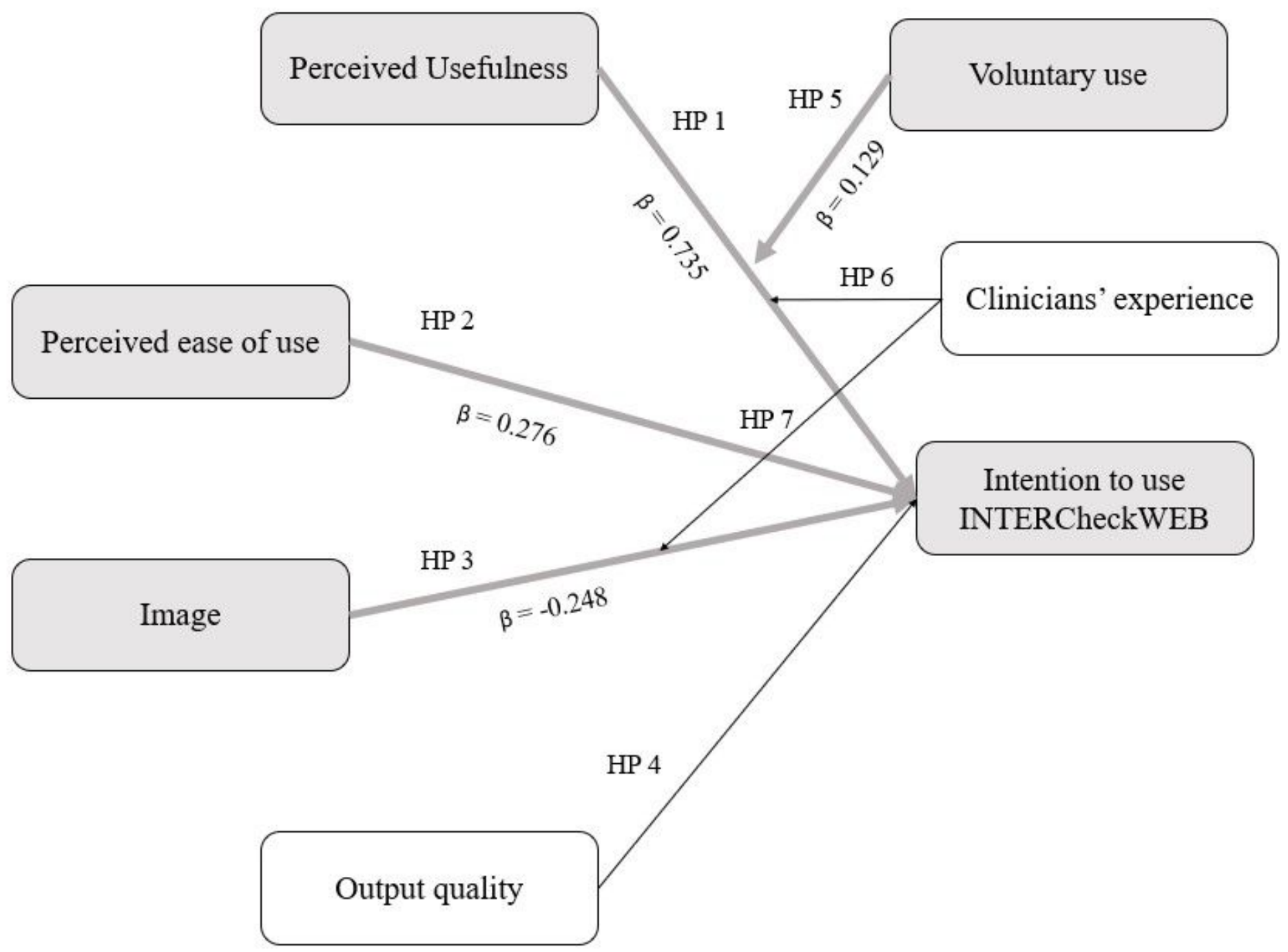

Figure 2

Framework with verified hypotheses

\section{Supplementary Files}

This is a list of supplementary files associated with this preprint. Click to download.

- SupplementaryFileQuestionnaire.docx 